\title{
It is time to reconsider the unnecessary division of premedical and medical studies
}

Young-Mee Lee

Department of Medical Education, Korea University College of Medicine, Seoul, Korea

The 2018 December issue of the Korean Journal of Medical Education posted an article about the history of medical education in Japan and the major issues raised during curricular changes [1]. In ancient times, Japanese medicine was primarily influenced by Korea and China. One of the first descriptions of medicine in Japan was in the 5th century, when traditional medicine was introduced by Koreans [2]. However, Japan opened up to the influence of western countries during 17th and 18th centuries, in advance of Korea and China. When the Meiji government was established, western medicine (which was dominated by the German system) was adopted in Japan; subsequently, the Japanese medical education system began to influence other Asian countries, including Korea and Taiwan. In the early 20th century, Japan established eight imperial medical colleges including Kyungsung University (Seoul, Korea) and Taipei National University (Taipei, Taiwan) [2]. These medical colleges required 2 years of premedical studies, followed by 4 years of medical studies. In some medical colleges, including those in Korea, this divided medical educational curriculum continues to the present day. It is time for a thoughtful discussion and careful assessment of whether separating medical education into these two distinct curricular systems is still effective and if there is a rationale for continuing it.

The problem with these distinct, separate education systems -2 years of premedical and 4 years of medical studies in Korea-is well known. During the premedical course of study, medical colleges provide general education appropriate for freshmen-level university students, including liberal arts, natural sciences, and some courses in preparation for medical studies. Premedical students are promoted to medical studies only after they complete the entire 2-year curriculum and earn the prerequisite credits. However, upon completion, the academic scores acquired during premedical studies do not influence medical students' future career paths, including applications for internships and residency training. This reduces study motivation and many students regard the premedical years as a time to relax or refresh after their highly competitive and stressful
Received: November 22, 2018 • Revised: November 23,2018 • Accepted: November 23, 2018 Corresponding Author: Young-Mee Lee (https://orcid.org/0000-0002-4685-9465) Department of Medical Education, Korea University College of Medicine, 73 Inchon-ro, Seongbuk-gu, Seoul 02841, Korea

Tel: +82.2.2286.1098 Fax: +82.2.928.1647 email: ymleehj@korea.ac.kr
Korean J Med Educ 2018 Dec; 30(4): 279-281. https://doi.org/10.3946/kjme.2018.102 eISSN: 2005-7288

(C) The Korean Society of Medical Education. All rights reserved. This is an open-access article distributed under the terms of the Creative Commons Attribution Non-Commercial License (http:// creativecommons.org/licenses/by-nc/3.0/), which permits unrestricted non-commercial use, distribution, and reproduction in any medium, provided the original work is properly cited. 
high school years. In addition, many students consider their premedical years as the last opportunity to enjoy their quality of life before the challenging times they will experience during their 4-year medical studies. The most serious adverse effect induced by these situation is that students' study habits can be ruined. Most students return to their conscientious and diligent study habits when promoted to the first year of medical studies, but some cannot overcome their decreased motivation to learn and continue to struggle with poor study habits. These students have difficulty adapting not only to the rigors of studying medicine but also to school life. Some of them flunk several times, which reduces their selfesteem and makes them unhealthy. The major issues with the current division of premedical and medical education systems in Korea can be summarized as: (1) lack of study motivation, (2) unhealthy study habits, and (3) lack in sense of identity as being medical students or future medical professionals [3,4].

For the last several decades, most medical education experts have stressed the importance of workplace-based experiential learning and recommend early clinical exposure right from the beginning of the first year of study. Through early clinical exposure, medical students can learn about people, patient suffering, cure and care, empathy and compassion, a doctor's role, other professionals' roles, and teamwork in real world practices. This experiential learning motivates medical students with an understanding of why they should study normal structures and functions of the body and pathophysiology, and how they can apply this fundamental knowledge to solve problems and help people reduce their suffering. In addition, students learn a physician's professional responsibility as well as the importance of cooperation and development of an understanding with allied healthcare professionals. The clear-cut separation of premedical and medical studies hinders not only workplace experiential learning in the early years but also the meaningful integration of basic, clinical medicine and medical humanities in real world contexts.

Japan, which influenced the establishment of western medical education systems in some Asian countries, underwent major reforms in its curricula in the 1990s. According to the article by Onishi [1], in 1991, the Ministry of Education of Japan issued the deregulation of the Standard for the Establishment of Universities with a few specific amendments. The amendments repealed the segmentation of general and specialized education, and the division between different general education subjects (such as the humanities, social studies, natural studies, foreign languages, and health and physical education). After this change, the system of premedical studies (Shingaku Katei) was abolished in the Faculties of Medicine and Dentistry [1]. The previously rigid way of calculating academic credits for each subject was made more flexible. As an example of this change, Nagoya University Medical College, which was one of the eight imperial universities established in the 1930s, now operates an integrated 6-year program with crossdepartmental subjects among science, liberal arts, and medical sciences [5]. Tokyo Women's Medical University provides a more integrated study experience to students through a 6-year medical curriculum [6]. The integrated 6-year medical curriculum is the common, basic medical education system used in other Asian medical schools as well. The University of Hong Kong provides organbased integrated courses, which combine all disciplines beginning with the first year after the completion of the 3-month course, 'Introduction to the art and science of medicine' [7]. The National University of Singapore (NUS) has a 5-year basic medical education system which consists of phase I \& II for clinical sciences, and phase III, IV, and V for clinical training. Parallel with this five-phase course of study, the NUS runs a lon- 
gitudinal track which encompass a clinical skills foundation and a patient-based program, health ethics, law, professionalism, and social medicine [8].

Medical education is a continuum running through basic medical education, postgraduate medical training, and continuous professional development. It is time to reconsider unnecessary divisions which hinder the effectiveness of medical learning, and achieving clinical competency and professional excellence. The division between premedical and medical studies in some Asian countries should be conscientiously discussed and judiciously assessed on the basis of its benefits and adverse effects on medical education. At least in some countries, including Korea, it is critical to break the 100-year long history of unnecessarily dividing premedical and medical studies. Medical schools must recognize the fundamental principle that medical education is a continuum.

ORCID:

Young-Mee Lee: https://orcid.org/0000-0002-4685-9465

Acknowledgements: None.

Funding: None.

Conflicts of interest: No potential conflict of interest relevant to this article was reported.

Author contributions: All work was done by Young-Mee Lee.

\section{References}

1. Onishi H. History of Japanese medical education. Korean J Med Educ. 2018;30(4):283-294.

2. Suzuki Y, Gibbs T, Fujisaki K. Medical education in Japan: a challenge to the healthcare system. Med Teach. 2008;30(9-10):846-850.

3. Lee YM, Lee KJ, Lee YJ, Ahn DS. Evaluation of integrated lectures at Korea University, College of Medicine. Korean J Med Educ. 2000;12(2):343-351.

4. Korea Association of Medical Colleges. KAMC guideline for premedical education. Seoul, Korea: Association of Medical Colleges; 2017.

5. Nagoya University Medical College. Curriculum. https:// www.med.nagoya-u.ac.jp/medical_/about/pdf/profile_me dical_2017_all.pdf. Accessed May 24, 2018.

6. Curriculum of Tokyo Women Medical University. School of medicine: curriculum. http://www.twmu.ac.jp/english/ e04d_curriculm.html. Accessed May 17, 2018.

7. The University of Hong Kong. 130 MBBS curriculum. https://www.med.hku.hk/programme/bachelor-of-medicin e-and-bachelor-of-surgery-programme-mbbs-130-curricul um. Accessed May 17, 2018.

8. Curriculum of National University of Singapore. Curriculum. http://nusmedicine.nus.edu.sg/admissions/medicine/ undergraduate. Accessed May 17, 2018. 